ÉGYPTE

monde arabe

\section{Égypte/Monde arabe}

4-5 | 2001

L'Égypte dans le siècle, 1901-2000

\title{
Du village au Caire, au village comme au Caire
}

Vers la métropole-État

\section{Eric Denis}

\section{(2) OpenEdition}

Journals

Édition électronique

URL : https://journals.openedition.org/ema/883

DOI : $10.4000 /$ ema.883

ISSN : 2090-7273

Éditeur

CEDEJ - Centre d'études et de documentation économiques juridiques et sociales

Édition imprimée

Date de publication : 30 juin 2001

Pagination : 225-253

ISBN : 2-87027-963-9

ISSN : $1110-5097$

Référence électronique

Eric Denis, « Du village au Caire, au village comme au Caire », Égypte/Monde arabe [En ligne], 4-5 | 2001, mis en ligne le 08 juillet 2008, consulté le 07 juillet 2022. URL : http://journals.openedition.org/ema/ 883 ; DOI : https://doi.org/10.4000/ema.883

Ce document a été généré automatiquement le 7 juillet 2022.

Tous droits réservés 


\title{
Du village au Caire, au village comme au Caire
}

\author{
Vers la métropole-État
}

\section{Eric Denis}

«Périssent les nations, la nature demeure » Du

$\mathrm{Fu}(712-770)$

1 L'Égypte est en voie d'urbanisation totale. «L'écouménopolis », conçu comme l'avenir de l'humanité par Doxiadis dans les années 1970, tendrait ici à se matérialiser l'écoumène devient une vaste agglomération ${ }^{1}$. Les populations et les activités de la Vallée et du Delta du Nil s'agglomèrent dans des unités de peuplement de plus en plus denses qui, par extension et coalescence, en viennent à ne plus former qu'une vaste tâche urbaine encore très ramifiée ${ }^{2}$. Telle est la conclusion d'un siècle marqué par un différentiel de croissance favorable au monde des villes. Je défends donc ici que tous les Égyptiens deviennent des citadins dans leur mode de vie, d'habiter et d'utiliser la vallée du Nil, étant entendu que l'Égypte, pour plus de $98 \%$ de ses habitants et sans grand espoir de changement, n'est toujours que la Vallée et le Delta, c'est-à-dire un espace plus dense que la mégalopolis nord-américaine avec plus de 1700 habitants par kilomètre carré3.

2 L'Égypte, à la fin du XIX siècle, était tout à fait comparable à un autre delta, les PaysBas : les surfaces cultivées étaient équivalentes de même que le nombre d'habitants. Les deux pays se classaient ainsi parmi les espaces habités les plus denses du monde, dépassés seulement par le Japon. À présent, les Pays-Bas comme le Japon constituent des archétypes de conurbations étendues à l'échelle d'un pays avec, entrelacée, une agriculture intensive à haut rendement. L'Égypte compte à présent 60 millions d'habitants contre 16 pour les Pays-Bas. Sur le siècle écoulé, la divergence radicale des démographies et l'inégale insertion dans l'économie-monde durant l'ère des empires et du colonialisme - l'Égypte colonisée s'opposant sur ce point aux Pays-Bas et au Japon ont largement contribué à la dissemblance des destins, notamment dans la consolidation des mondes urbains. 
3 Pour autant, les niveaux d'agglomération des populations et, par extension, d'urbanisation sont convergents. Désormais, en effet, près de 70 \% des Égyptiens vivent dans des agglomérations de plus de 10000 habitants, qui participent d'une forme de plus en plus globalisée de peuplement tout en étant travaillées par les traces et la volonté de réappropriation de l'histoire locale. La rencontre des temporalités et des lieux ou des échelles et des moments compose, ici comme ailleurs, la complexité des relations entre espace et société, même dans un contexte incontestable d'interdépendance généralisée. L'histoire et la géographie ou le jeu des scansions et des territoires ne s'arrêtent pas.

4 Ce prisme urbain amène à concevoir un écoumène égyptien plus imbriqué qu'il n'est généralement conçu ou officiellement découpé, c'est-à-dire à admettre une profonde intrication des univers ruraux et urbains. Cela nous renvoie à l'étroite corrélation des densités des campagnes et des villes dans les grands deltas ; toutes deux sont extrêmes, soulignant bien l'indéfectible relation. La ville se façonne à l'image de la campagne environnante et des modes de production agricole. Ce qui, au passage, contredit la supposée indépendance croissante des milieux urbains par rapport à leur environnement proche, de même que l'idée convenue d'une dépendance accrue et surdéterminante à l'égard des flux transnationaux. La trace joue ici un rôle très fort et contraignant. L'agglomération cairote est neuf fois plus dense que celle de Paris (25000 $\mathrm{hab} / \mathrm{km}^{2}$ ) et cette densification à l'extrême, que l'on ne retrouverait qu'en Asie, concerne tous les échelons du peuplement, depuis le hameau. Ce qui doit nous conduire à l'idée que le procès d'urbanisation n'est pas réductible à la mégapolisation. Certes, en un siècle, Le Caire s'est accru 18 fois, passant de 600000 habitants à 11 millions mais, dans le même temps, la population urbanisée en dehors de la capitale a été multipliée par près de 22, passant de 1,4 millions à 30,5.

5 Il faut encore accepter, pour nous suivre, la notion de "villes de citadins des champs ", telle que la propose Max Weber. De même, qu'il faut prendre au sérieux la grille d'analyse proposée par le géographe Gamâl Hamdân dans son monumental ouvrage, La personnalité de l'Égypte, notamment quand il explique que «l'urbanité commence à l'échelle du village » ou que « le village est l'instrument de mesure du changement » ${ }^{4}$.

6 Dans cet état d'esprit, nous pouvons entreprendre une réflexion sur la genèse, les modalités et les rythmes du procès d'agglomération qui a conduit à l'urbanisation généralisée de l'Égypte, sans se laisser abuser par les spectres trompeurs associés à l'apparent gigantisme cairote ou aux mirages de la fertilisation et du peuplement des déserts inspirés par l'immensité inexploitée, mais largement inexploitable ou alors, dans des coûts inacceptables. En ce sens, comprendre comment s'y cristallisent en agglomérations les modes d'habiter, de travailler, de circuler et d'échanger nous paraît essentiel. Donner à voir et expliquer les rythmes et les modalités de transformation du système de peuplement, ses bifurcations, invite à regarder l'histoire sociale et politique de l'Égypte contemporaine sous un angle différent, non limité par l'écran de la construction nationale, de la communauté forcément totalisante, mais ancré dans la matérialité des constructions territoriales infranationales et des luttes de représentation. L'idée d'urbanisation totale est, elle-même, une apparence morphologique qui masque l'essentiel, c'est-à-dire l'affirmation au cours du siècle d'une métropole-État et un mode métropolisé, polarisé par la capitale, d'exploitation d'un territoire conçu comme un arrière-pays et un pays arriéré. 
7 Tel est le cœur de la thèse qui guide la lecture à venir d'un siècle de métropolisation globale plutôt que d'épanouissement d'une démocratie citadine. L'« écouménopolis ", en effet, au sens de cité globale, est loin de s'épanouir en Égypte, tant durant le siècle écoulé que dans l'actualité la plus immédiate. Bien davantage, la généralisation de la condition urbaine s'accompagne d'une "délibéralisation" politique, pour le moins d'une "non-démocratisation", et d'une administration policière centralisée du territoire et des villes, justifiée par le nécessité de protéger le développement matériel de la Nation, elle-même conçue comme une totalité sans nuance territoriale autre que la centralisation comme l'a construite Gamâl Hamdân. La lecture de l'urbanisation proposée ici vise à montrer que la diffusion jusqu’à la coalescence générale du système de peuplement n'est pas synonyme d'une décentralisation mais, bien au contraire, participe d'un mode de gouvernement centralisé, régnant sans partage, organisant la dépendance à la capitale vers qui sont drainées, selon une logique de colonisation intérieure, richesses produites, compétences et rentes. En cela le modèle de la métropole-État post-nationaliste ne diffère pas ou, plutôt, reprend, les instruments du colonialisme auquel le coup d'État des Officiers libres était supposé mettre un terme au profit de la communauté nationale. Les outils techniques du contrôle et de l'exploitation coloniale du territoire et du peuple ont été « internalisés », repris dans la mise en place de l'appareil d'État et mis au service d'un projet impérialiste, au sens de domination interne et d'affirmation sur la scène internationale; deux faces d'un même projet qui suppose une métropole concentrant et organisant la puissance publique, polarisant les initiatives privées tout en les contrôlant, alors qu'elle favorise l'atomisation et la dépendance, donc le contrôle, des citadins ordinaires devenant peu à peu l'écrasante majorité des citoyens égyptiens.

La capitale : la concordance des temporalités urbaines et politiques

$8 \quad$ Le XXe siècle est sans doute le temps de l'affirmation d'une Égypte indépendante qui s'impose comme un État-nation à la charnière de plusieurs mondes - guide des pays non-alignés et de l'unité du monde arabe, porte-parole du monde musulman, frèreennemi indéfectible des États-Unis ou encore partenaire privilégié des Européens sur la rive sud de la Méditerranée et puissance respectée sur la scène africaine. La construction de cette image de puissance régionale incontournable s'appuie sur la masse démographique et, en particulier, sur l'onde de polarisation autour de la capitale égyptienne amorcée par un différentiel d'attractivité et des crises agricoles bien antérieures au coup d'État.

9 La date charnière de l'histoire politique contemporaine, 1952, correspond à une phase d'inflexion de la croissance extrême de la capitale, donc à la fin de la captation exclusive d'une large part du flux migratoire depuis les provinces les plus exportatrices de mains-d'œuvre, comme la Minûfiyya dans le centre-Delta ou Suhag en Haute-Égypte (Ireton, 1998a). Dès lors, le différentiel de croissance démographique ne va cesser de fléchir au profit d'agglomérations de plus en plus petites et provinciales indiquant une redistribution structurelle des populations et des activités (graph. 1) avec, bien entendu, un décalage et une inertie entre les tendances observées en termes de taux et les évolutions perceptibles en valeur absolue. Mais sur le siècle, il y a bien concomitance des inflexions démographiques et politiques à mi-parcours. Avec le jeune régime, la polarisation devient de plus en plus qualitative, davantage économique, politique et culturelle que démographique. L'attractivité devient sélective, le 
mouvement de métropolisation s'enclenche; la polarisation et l'organisation des flux accompagnent le drainage des richesses.

Graphique 1 : L'onde longue de métropolisation (1897-1996) - Taux annuel de croissance démographique par taille d'agglomérations (en milliers d'habitants)*

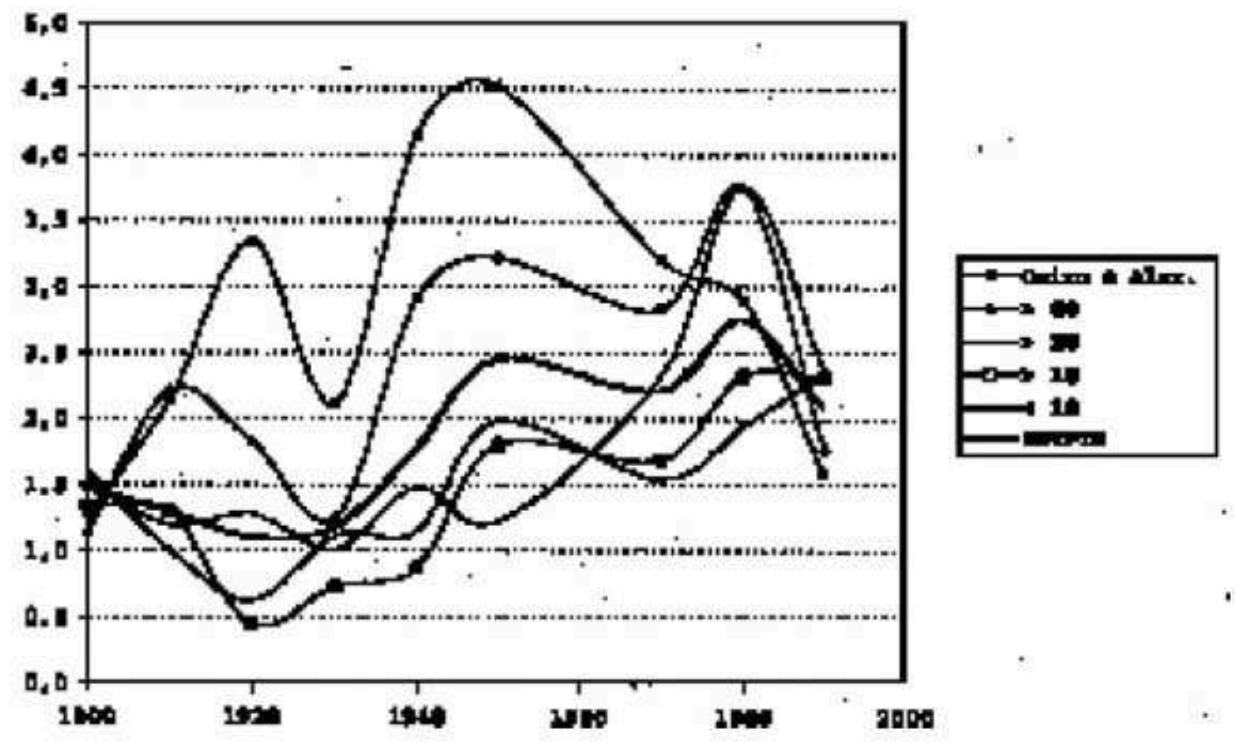

* Les agglomérations sont considérées rétrospectivement dans leurs limites de 1996, les unités géographiques sont donc constantes sur la période 1897-1996.

10 Toutefois, si la tendance est bien là synchroniquement, en valeur absolue, l'agglomération cairote, à limite constante et dans sa plus grande extension de 1996, ne connaîtra un fléchissement de sa croissance que dans les années 1990 augmentera de 2 millions d'habitants entre 1976 et 1986 - un maximum -, puis encore d'un million durant la décennie suivante. Autrement dit, les 50 dernières années ont été marquées par l'impérative nécessité d'équiper et d'assurer le fonctionnement d'une capitale toujours débordée par une forte pression démographique initiée par les tendances migratoires du début du siècle.

11 Enfin indépendante, la jeune nation est héritière d'une dynamique antérieure qu'elle doit organiser jusqu'à ce jour. Ainsi s'imposent les conditions d'un biais mégapolitain durable, renforcé par la nécessité d'affirmer, pour un régime à la recherche d'un leadership panarabe et tiers-mondiste, le statut de capitale régionale du Caire ${ }^{5}$. Elle capte en effet une large part de l'effort industriel, avec les aciéries et les cimenteries de Hilwân ou les usines militaires du nord de l'agglomération, et des investissements en faveur de la construction, avec les programmes de logements publics et ceux destinés à accueillir les services d'un appareil d'État qui renforce sa technostructure. Puis s'imposent, afin d'entretenir et de développer ce potentiel de production et de commandement, d'énormes chantiers d'infrastructures - ponts, autoponts, égouts et métro (Denis, 2001).

L'onde de très forte croissance des plus grandes villes est aussi à comprendre comme un facteur de divergence des destins socio-démographiques entre villes et campagnes comme entre régions. Jusqu'au début du XXe siècle, les différences sont minimes : les villes n'attirent pas de ruraux, les croissances sont comparables. Ensuite, le différentiel se creuse profondément au profit des plus grandes villes, au premier rang desquelles Le 
Caire. À la fin du siècle, les rythmes de croissance convergent à nouveau, signe non plus d'une faible distinction des villes de l'horizon des campagnes, mais davantage de la diffusion de la condition urbaine à l'ensemble du pays. Pour autant, il n'y a pas de convergence des modes de faire ville car, entre-temps, une exfiltration qualitative s'est opérée au sein du système de villes. Le système de peuplement, tout en devenant système de villes, est resté très hiérarchisé.

Déjà, la métropole redistribue ses habitants en fonction de leurs revenus, après les avoir rendus totalement dépendants du monde urbain, de ses productions, de ses services et de son administration. La capitalisation métropolitaine des fonctions de commandement publiques et privées, comme des activités à plus forte valeur ajoutée, renforce encore le processus de redistribution sociale centrifuge en accroissant la pression foncière sur un parc de logements et de locaux d'activités déjà fortement contraint par le poids de la propriété publique hors marché qui domine le paysage métropolitain, surtout après les nationalisations de 1952-1956.

1900-1950 : d'un monde de villes et d'agriculture dépendant mais décentralisé à un état métropoliséL'industrialisation de l'agriculture et l'affirmation du système de villes

Durant tout le XIX ${ }^{e}$ siècle et jusqu'aux années 1910, le poids du Caire dans la population de l'Égypte demeure extrêmement stable et faible. En 1848, il était de 5,9 \% et, en 1907, de 5,5\%. Mais il double ensuite en 40 ans, puis double encore durant les 40 ans suivants, pour culminer à 19,3 \% en 1986 et retomber à $17 \%$ en 1996 (dernière borne censitaire dont nous disposons). La fin du XIX ${ }^{e}$ siècle est marquée par la création de villes nouvelles, qui ouvrent la carte de l'Égypte utile. Les créations pures, comme les villes du Canal, Zaqâzîq ou Kafr al-Shaykh, ou les villes sucrières du Sacîd, comme al-Fikriyya, Armant ou Kûm Umbû, ne sont pas favorables à une plus grande polarisation autour du Caire. De plus, ces fondations s'accompagnent d'un élargissement des surfaces cultivées, tant sur les marges désertiques qu'en aval du Delta, du fait d'importants travaux de drainage. En 1882, l'Égypte compte 5000 'izba-s, nouveaux hameaux créés depuis moins de 50 ans et regroupant la main-d'œuvre des grands domaines agricoles, distribués pour l'essentiel sur les marges du Delta. Ces investissements contribuent à la redistribution centrifuge des populations et à la constitution de pôles secondaires de peuplement là où s'implantent des filières agro-industrielles cotonnières ou sucrières. L'essor des villes commerciales littorales, d'Alexandrie et des villes du Canal, qui accompagne l'extraversion de l'économie et l'inscription de l'Égypte parmi les exportateurs tropicaux, renforce encore l'ampleur de la tendance (Alleaume \& Denis, 1998).

15 Si Le Caire dispose déjà de la plupart des fonctions de commandement politique, il partage encore le pouvoir financier avec les grandes banques d'Alexandrie. Surtout, des formes émergeantes d'édilité commencent à assurer la gestion des grandes villes de province, du Delta notamment (Delcroix, 1922). Les créations de municipalités à la fin du XIX ${ }^{e}$ siècle - Alexandrie en 1890, Mansoura en 1896, plus d'une trentaine de villes secondaires ensuite -, témoignent de la consécration d'une économie multipolaire et d'une décentralisation des compétences, après la période de mise en place d'un appareil d'État centralisé sous le règne de Muhammad cAlî. L'influence des marchands étrangers clairement et légalement sur-représentés dans ces conseils municipaux n'est bien entendu pas étrangère à l'émergence de ces aspirations locales, mais au-delà, l'administration décentralisée des villes secondaires constitue bien un moment de 
maturation du système politique qui va se déliter ensuite au profit d'une recentralisation poursuivie jusqu'à présent sous couvert de construction nationale.

Dans ce contexte de constitution de polarités secondaires, il n'y a pas de place pour une croissance différentielle du Caire. La capitale n'attire pas particulièrement les migrants des provinces. Le XIXe siècle est même parcouru par une angoisse démographique qui nous semble aujourd'hui incongrue. L'Égypte, en effet, avait peur de manquer de maind'œuvre, notamment agricole, mais aussi pour ses manufactures et son armée, tant et si bien qu'en 1860 il fut même projeté d'avoir recours à l'immigration (Owen, 1981). Mais l'inquiétude s'estompe avec l'emballement démographique de la seconde moitié du XIX siècle (la population croît de $50 \%$ entre 1870 et 1900) et l'achèvement du barrage à l'apex du Delta en 1890. Il permet en effet de réduire les besoins en main-d'œuvre pour l'entretien saisonnier du réseau d'irrigation et autorise une pratique étendue de la double, voire de la triple récolte. Au total, entre l'augmentation des rendements et les bonifications, la surface récoltée augmente de $60 \%$ durant les 30 dernières années du XIX ${ }^{e}$ siècle. L'abolition de la corvée en 1892 bénéficie de ces conditions devenues favorables à un marché libre du travail ; bientôt même, un surplus de main-d'œuvre agraire se fait jour et vient nourrir l'onde de croissance métropolitaine.

Crises agricoles, exode rural et métropolisation

La puissante dynamique agro-industrielle semble alors en mesure d'absorber la croissance démographique et de nourrir les villes. Mais, au début du siècle, l'économie agricole s'essouffle, les rendements stagnent, voire baissent, et, à partir des années vingt, les surfaces en culture ne s'étendent plus. La surface récoltée ne croît que de 10 \% entre 1907 et 1937, malgré l'emploi devenu massif d'engrais chimiques, pratiquement inconnus à la fin du XIX siècle ${ }^{6}$.

Il faudra attendre la période postérieure à 1952 pour que de nouvelles bonifications extensives permettent un redéploiement de l'économie agricole et les années 1970-1980 pour que les rendements s'accroissent à nouveau très sensiblement. La décomposition du PNB pour la période 1940-1950 indique clairement que la croissance annuelle du produit agricole est nulle alors que celle de l'industrie et des services dépasse en moyenne $6 \%$ par an après la dépression des années 1930 (Mead, 1967). Jusqu'au début des années 1940, la création d'emplois dans l'agriculture reste élevée, se redressant même durant la crise des années 1930, mais elle s'effondre ensuite. Cette profonde dépression s'explique par la réorganisation à l'échelle mondiale du secteur textile et la baisse des cours du coton. Les activités manufacturières égyptiennes déclinent, perdant même des emplois (-0,9 \% par an entre 1927 et 1937). L'agriculture, pourtant elle-même sinistrée du fait de l'extrême dépendance à l'exportation qu'elle doit à l'importance prise par la culture du coton, agit alors comme secteur refuge. Pour le moins, l'économie urbaine n'attire pas. Ainsi s'explique le creux dans l'onde de métropolisation (graphique 1). Durant la période intercensitaire 1927-1937 et dans un contexte global de très faible croissance de l'emploi $(1 \%$ par an pour une croissance démographique de 1,2 soit le plus mauvais rapport du siècle), c'est encore dans l'agriculture que se créent $80 \%$ des emplois.

19 Mais la reprise est simplement conjoncturelle et la bifurcation vers les secteurs manufacturiers et surtout les services s'impose encore plus brutalement dans les années 1940. Les taux de croissance annuelle atteignent alors respectivement 3,6 \% et $3,8 \%$, tandis que celui de l'agriculture tombe nettement en dessous de $1 \%$, pour un taux de croissance général de la population de 1,8. Il se crée désormais autant d'emplois 
dans les services qu'il s'en créait auparavant dans l'agriculture : 40000 contre 15000 dans l'industrie. L'explosion $\mathrm{du}$ secteur des services témoigne d'une phase d'urbanisation extrêmement rapide, plus rapide que celle de l'appareil de production. Durant cette période, un véritable exode rural alimente les grandes métropoles, avec des modes d'insertion qui relèvent pour une large part de l'auto-emploi et de la précarité (journaliers, vendeurs ambulants, etc.). Dans la phase d'accélération extrême du rythme de croissance des métropoles, entre 1937 et 1947 (plus de $4 \%$ par an contre 2 durant la décennie antérieure), la population des gouvernorats urbains, du Caire, d'Alexandrie et des trois villes du Canal, augmentent de $52 \%$ alors que, dans les autres provinces, le gain n'est que de $14 \%$. En faisant l'hypothèse d'une croissance naturelle identique dans les deux zones, l'écart suppose la migration de plus de 760000 personnes durant la période, soit $5 \%$ de la population de 1937 qui se seraient déplacés vers les grandes métropoles en dix ans. Pour cette même période, Mead estime à $25 \%$ du total de la main-d'œuvre adulte mâle le glissement vers les gouvernorats urbains. De façon certaine, c'est-à-dire de source censitaire, en 1937, 667000 habitants de ces 5 villes n'y étaient pas nés; ils seront plus d'un million en 1947. En 1947 toujours, ces métropoles concentraient $64 \%$ des Égyptiens ayant effectué au moins une migration interprovinciale, contre $37 \%$ en 1907. Le Caire, à lui seul, en regroupait $41 \%$, contre 24 \% quarante ans plutôt (Ireton, 1998b).

$\mathrm{Si}$, pour une large part, la croissance très forte des services témoigne d'une surpopulation conjoncturelle, donc d'un trop fort appel des métropoles, l'attractivité des plus grandes villes fait système avec l'amorce d'une bifurcation structurelle de l'appareil productif, avec l'industrialisation à forte intensité de main-d'œuvre. Surtout, le trop plein relatif, peu à peu, génère ses propres activités; l'économie informelle se développe, répondant aux besoins de logement, d'équipement ménager, d'approvisionnement quotidien ou de déplacement du plus grand nombre. La forme urbaine s'en trouve radicalement transformée. La ville ordinaire devient para-légale et sous-équipée du fait de sa non reconnaissance par l'institution planificatrice qui se met en place.

21 Certes, l'effort pour mettre à disposition un habitat social collectif s'accroît dans les années 1960, accompagnant notamment la création des combinats industriels et la fidélisation au régime des fonctionnaires et des militaires, mais le caractère illégal de ce qui loge majoritairement les citadins est parallèlement amplifié par la publication de normes de plus en plus restrictives, notamment en matière de « constructibilité » des réserves foncières péri-urbaines et, en particulier, des terres agricoles, mais aussi des déserts. Le régime, s'appuyant sur la planification, reprend et institutionnalise les idées du réformisme social portées, avant 1952, par des élites éclairées et prétend couvrir et organiser l'ensemble des besoins des citoyens ${ }^{7}$. Ce faisant, dans un contexte de pénurie et donc d'incapacité à fournir, il a contribué à élargir l'illégalité de la promotion immobilière couvrant les besoins du plus grand nombre. Organisant le manque, il a progressivement, par exclusion, définit les contours d'une société majoritairement dépendante et sans droit, réduite à une citadinité de seconde zone. Une clientèle obligée a ainsi été socialement bornée et géographiquement localisée, créant par làmême de multiples fonctions d'intermédiaires exploitant la frontière entre légalité et illégalité et servant de passeur entre les administrations, les habitants et les microentrepreneurs. Par leur médiation, ils entretiennent clientélisme et dépendance. 
Quand la mégapolisation appelle un nouveau gouvernement de l'ÉgypteAppauvrissement et captation des revenus par la métropole

L'accélération de la croissance métropolitaine durant le second quart du XX'siècle n'est pas le produit d'une croissance industrielle qui aurait tardé à advenir. Entre 1937 et 1945, la part de l'industrie dans le produit intérieur brut passe simplement de 8 à $11 \%$, alors que celle de l'agriculture baisse à peine, passant de 49 à $44 \%$. Ces chiffres montrent en creux que les services, en passant de 43 à $45 \%$, restent peu productifs et absorbent en masse une main-d'œuvre qui cherche à s'insérer en ville, dans les plus grandes métropoles en particulier (Mabro, \& Radwan, 1973).

La population active fuit surtout l'appauvrissement des campagnes, l'augmentation du loyer de la terre, comme l'impossible accès au crédit et la concentration inexorable des terres agricoles. Si, jusque dans les années 1930, le secteur agricole, malgré ses piètres performances, fixe encore des populations appauvries, l'absence de redistribution vers les campagnes et l'investissement dans les plus grandes villes des plus-values mettent finalement la main-d'œuvre en mouvement. Le contexte est à l'appauvrissement général, avec un produit national par habitant qui ne peut se maintenir du simple fait du rythme d'accroissement de la population; il baisse dans les années 1920, puis remonte de $0,25 \%$ durant les années 1930 , pour perdre à nouveau $5 \%$ pendant la Seconde Guerre mondiale et remonter sensiblement ensuite. Il n'y a donc pas d'élévation du niveau de vie durant près de 50 ans. Pire même, compte tenu des inégalités de répartition, l'appauvrissement du plus grand nombre peut être clairement relevé. Entre 1929 et 1937, la consommation de céréales baisse de $12 \%$, celle de tabac de $30 \%$ et celle de sucre de $40 \%$ (Hansen, 1965), alors que les maladies endémiques, comme la malaria, connaissent une recrudescence, notamment en Haute-Égypte. Les salaires agricoles baissent de 1920 à 1940, alors que ceux de l'industrie croissent irrésistiblement (jusqu'à $20 \%$ durant la Seconde Guerre mondiale). Ils sont entre 2,5 et 3 fois plus élevés que ceux de l'agriculture, incitant, là encore, les paysans sans terre et les journaliers à tenter leur chance en ville. D'autant que, si l'essor industriel reste très faible et le secteur marqué par la petite taille de ses ateliers, avec la dépréciation à répétition de la valeur du coton, une partie des grands propriétaires terriens s'orientent néanmoins vers l'industrie. Des grands établissements apparaissent donc entre les deux guerres mondiales.

La mise en mouvement de la main-d'œuvre est encore favorisée par le caractère saisonnier tant du travail agricole, avec le pic des récoltes, que d'une part importante des emplois industriels. Une usine sucrière comme celle de Hawâmdiyya, au sud du Caire, pouvait dès le début du siècle employer jusqu'à 15000 personnes durant les campagnes de production suivant les récoltes de la canne à sucre. Ainsi se constitue progressivement une population agro-ouvrière mobile, à la charnière entre la campagne et la ville. Elle est sans doute l'une des premières à développer des compétences lui permettant de s'établir de manière plus définitive en ville et à transformer l'univers urbain. Simple indice, fin 1950, l'usine de Hawâmdiyya est le théâtre d'une des premières grèves à laquelle ont pris part 4000 ouvriers. Elle annonce les événements qui conduiront à l'intensification et l'extension des mouvements de grève dans le secteur textile, à Shubrâ al-Khayma notamment, mais aussi dans les transports urbains, jusqu'au basculement radical de 1952.

Industrialisation, désindustrialisation et révolution nationale 

d'une industrie agroalimentaire structurant de nombreuses villes et bourgs et leur arrière-pays, la mise au travail de la main-d'œuvre, sous-employée à grande échelle, par les forces alliées durant la Seconde Guerre mondiale jouent un rôle essentiel dans l'accélération du processus de métropolisation. En 1943, elles emploient 260000 personnes, des migrants récents qui, une fois « démobilisés » et en l'absence de terres disponibles, ne retournent pas vers leur campagne d'origine (Beinin \& Lockman, 1987). En 1946, avec le départ des forces alliées, non seulement une population ouvrière qualifiée naissante se retrouve sans emploi, mais tout un monde de services, d'importateurs et de commerçants voit son activité décliner. Un sous-prolétariat urbain émerge ainsi de façon massive sur le devant de la scène sociale. Entre 1947 et 1960, l'emploi industriel ne croît plus que de 2,2 \% par an et les services de 2,4 \% contre, respectivement 3,6 et 3,8 durant la décennie 1937-1947 . L'Égypte n'est pas en mesure de capitaliser l'expérience industrielle liée à la délocalisation de l'économie de guerre des forces alliées : les ateliers sont démantelés et les compétences largement perdues. Une large part de la main-d'œuvre urbaine rejoint la frange des journaliers, de la maind'œuvre flottante qui souffre de la quasi-désindustrialisation de l'immédiat aprèsguerre. Puis, si l'investissement industriel ne fléchit pas, l'emploi purement industriel stagne, voire décline ${ }^{9}$. L'accroissement de la productivité, une utilisation rationalisée de la main-d'œuvre associée à une mécanisation accrue et à la création d'établissements plus grands contribuent à la non-absorption de la main-d'œuvre ouvrière disponible, alors que la demande intérieure stagne. La période est donc marquée par une mobilisation du capital disponible par le secteur industriel, mais sans création d'emplois. Ainsi, les manifestations que les dirigeants interprètent ou tentent de réduire à un mécontentement grandissant contre l'occupation anglaise est, aussi, le produit d'un sous-emploi croissant. En partie donc, l'apparente surpopulation, produit d'un exode rural sans relation avec la dynamique de l'emploi - raccourci simpliste servant de prêt-à-penser, ici comme ailleurs, pour qualifier les conditions brutales d'émergence des mégapoles des pays du Sud - se voit contredite par cette tendance postérieure à la Seconde Guerre mondiale, caractérisée par la rupture de l'appel de main-d'œuvre amorcé antérieurement. L'économie urbanisée, métropolisée pour l'essentiel, a produit en elle-même et dans ses rapports, voire sa dépendance, aux instabilités économiques et politiques mondiales les conditions d'émergence d'un sousprolétariat urbain composé de journaliers sous-employés et d'un secteur tertiaire à faible productivité.

Cette combinaison de la métropolisation de la croissance démographique et de la stagnation de la création d'emplois productifs contribue largement à l'intensification des mouvements de grève et à la contestation violente du pouvoir, qui trouve un exutoire emblématique dans l'incendie du centre d'affaires du Caire en janvier 1952. Le mouvement communiste et surtout les Frères musulmans savent catalyser et conférer un sens politique à ce mécontentement, qui ne vise pas seulement les excès et le désintérêt du Roi, comme sa responsabilité dans la défaite en Palestine, ou encore l'occupant anglais, mais aussi, voire surtout, le parti Wafd. Ce dernier, en effet, représente d'abord les intérêts des grands propriétaires terriens enclins à une exploitation spéculative et financière de l'investissement urbain et industriel. Le mouvement populaire des années 1950 est donc aussi, et peut-être avant tout, le produit du basculement du centre de gravité démographique des campagnes vers les villes, alors que la représentation du pays demeure centrée sur les valeurs de la terre. 
27 À partir de 1952, le pouvoir politique affirme un intérêt grandissant pour le peuple des villes: il en va de la stabilité de la Nation. Mais, longtemps, le monde des villes se réduira au Caire et très secondairement à Alexandrie. La cité méditerranéenne perd en effet beaucoup de ses compétences au profit de la capitale où se concentre le pouvoir. Elle se voit privée de toute autonomie financière et décisionnelle, alors que le centralisme administratif s'impose. Le renforcement de son tissu industriel sur ses marges, alors que son centre se délite faute de renouveau des fonctions de commandement, conduit à la fragmentation du tissu urbain alexandrin. Par ailleurs, les politiques d'équipement à destination des villes de province demeurent très réduites, alors que les conflits sur les rives du Canal de Suez grèvent durablement le potentiel de croissance de ses villes ${ }^{10}$.

Faire capitale mais ne pas voir et refuser l'inévitable débordementPlanifier, moderniser et contenir

Après le coup d'état des Officiers libres, la modernisation de la capitale s'impose sans détour ; elle doit en effet être en mesure de porter un projet de société qui suppose la mise en place d'un puissant appareil d'État centralisé et la concentration des moyens de production. Dès 1956, conçu par de jeunes ingénieurs formés à Londres, le premier schéma directeur ébauche l'intégration de la région métropolitaine. Ses idées fortes, pas encore mises en œuvre faute de moyens, seront reprises dans le second schéma de 1971/1974.

Il invite à la constitution d'un pôle administratif et gouvernemental sur le plateau désertique; ce sera Madinat Nasr, à proximité de la présidence. Ce quartier neuf compte aujourd'hui plus de 600000 habitants, un parc de logements neufs inhabités en mesure d'en accueillir le double et de nombreuses institutions publiques civiles et militaires. Des ministères s'y relocalisent encore, comme le nouveau siège du ministère des Finances en voie d'achèvement. Par ailleurs, le schéma de 1956 propose la constitution, au sud du Caire, d'un puissant conglomérat industriel associant intérêts civils et militaires. Sa mise en place pendant dix ans, autour de l'acier, du ciment, de la chimie et de l'automobile, absorbera près de la moitié des capacités publiques d'investissement industriel. Il programme encore le renforcement du centre textile hérité de Shubrâ al-Khayma et le développement de pôles agroalimentaires à Imbâba et Gîza. Outre que ce plan polarise l'effort d'industrialisation à proximité immédiate de la capitale, il s'agit toujours d'un projet de modernisation à forte intensité de capital, donc pas forcément le plus pertinent en matière d'emplois. Surtout, il amorce l'urbanisation de riches terres agricoles que les administrations compétentes chercheront et cherchent encore à contenir, notamment sur la rive ouest du Nil, ou encore au nord, en Qalyûbiyya.

Ces principes ont pour objet, d'une part, la préservation de l'intégrité du Caire, donc la satellisation des flux de migrants à proximité des nouveaux pôles industriels et, d'autre part, la fondation sur terrain vierge d'un cadre bâti fonctionnel et résolument moderniste. Ce dernier doit réfléchir une image du gouvernement égyptien en rupture radicale avec l'Égypte des palais et des villas et afficher les capacités de la filière de la construction, désormais industrialisée et appuyée sur une offre d'acier et de ciment en pleine expansion. Il faut le concevoir comme la réinsertion de l'Égypte par sa capitale dans la dynamique de croissance urbaine qu'ont connue les démocraties industrielles et le bloc soviétique après la Seconde Guerre mondiale. L'esprit moderniste, c'est-à-dire la production industrialisée de logements standardisés, est alors universellement au cœur 
des modalités d'intégration et de renouvellement des cités, du passage d'un monde de villes à un monde urbain.

31 Les schémas directeurs suivants, ceux de 1974 et de 1982, reprennent ces thèmes, ajoutant simplement un boulevard périphérique qui marque parfaitement, à l'image d'une enceinte, la limite de la ville dont l'intégrité doit être préservée et des villes nouvelles qui prolongent le projet réformiste de refondation de la société urbaine sur terrain vierge, combiné à l'idée de report de la croissance sur les plateaux désertiques afin de protéger les terres agricoles (El Kadi, 1990). Dès lors, les politiques urbaines cherchent à contrecarrer les effets induits par les orientations des années 1960 qui ont permis les premières extensions, notamment sur la rive ouest - industrielles mais aussi résidentielles avec Muhandisîn notamment. En fait, les cités ouvrières d'Imbâba avaient ouvert la voie avant 1952. Le premier plan, celui de 1956, conçoit la région métropolitaine comme l'Égypte en réduction et, donc, la satellisation industrielle comme une politique de lutte contre les migrations à destination du Caire proprement dit. Simplement, la segmentation de l'offre immobilière impose que le centre ancien et les vieux faubourgs avec leur parc de logements dégradés restent pour longtemps encore les points d'insertion des migrants des provinces. Ils y apprennent la ville et capitalisent compétences et moyens d'accéder au logement, avant qu'eux ou leurs enfants trouvent à se loger sur les marges industrielles et populaires désormais intégrées à l'agglomération. L'accès aux nouvelles périphéries exigent des compétences urbaines dont ne disposent pas les primo-migrants des provinces. L'apprentissage se fait au cœur de la ville et dans les faubourgs anciens qui conjuguent à la fois les métiers peu qualifiés de service, de portefaix et de manœuvre et un habitat dégradé accessible. La dé-densification au profit des périphéries n'interviendra qu'avec le tarissement progressif du flux de migrants provinciaux. Et ceci, sans compter l'importance croissante, parmi ces migrants, des habitants qualifiés des villes secondaires qui trouvent immédiatement à s'insérer dans le tissu des activités manufacturières périphériques.

Une planification débordée

Si ce plan est conçu pour faire du Caire une capitale à l'image des plus grandes métropoles du monde, il ne prend pas la mesure de ce que sera Le Caire quelques 40 ans plus tard. Il veut faire du Caire une métropole internationale, alors qu'elle devient une mégapole. Il prévoit 5,5 millions d'habitants en l'an 2000, alors qu'elle en compte à présent plus du double et que, dès 1968 , elle dépasse les 5 millions d'habitants. Ce n'est pas que les politiques mises en œuvre soient sans effets, mais les conséquences du puissant mouvement migratoire antérieur sont sous-estimées.

En 1907, 24 \% des habitants du Caire n'y sont pas nés, ils seront plus de $35 \%$ en 1960, mais dès 1976 plus que $26 \%$ et, enfin, moins de $12 \%$ en 1996 (graph. 2). Ainsi, dès le début de la période nassérienne, les migrations à destination de la capitale fléchissent, mais l'apport de la première moitié du siècle a déjà radicalement transformé la structure démographique de la capitale. Elle est rajeunie par l'apport migratoire qui retarde la transition démographique. Ensuite, les enfants des migrants commencent à chercher à se loger ; ils augmentent la pression sur le marché immobilier et poussent à l'ouverture de nouveaux fronts d'urbanisation. 
Graphique 2 : la part des non-natifs du Caire dans la population du Caire

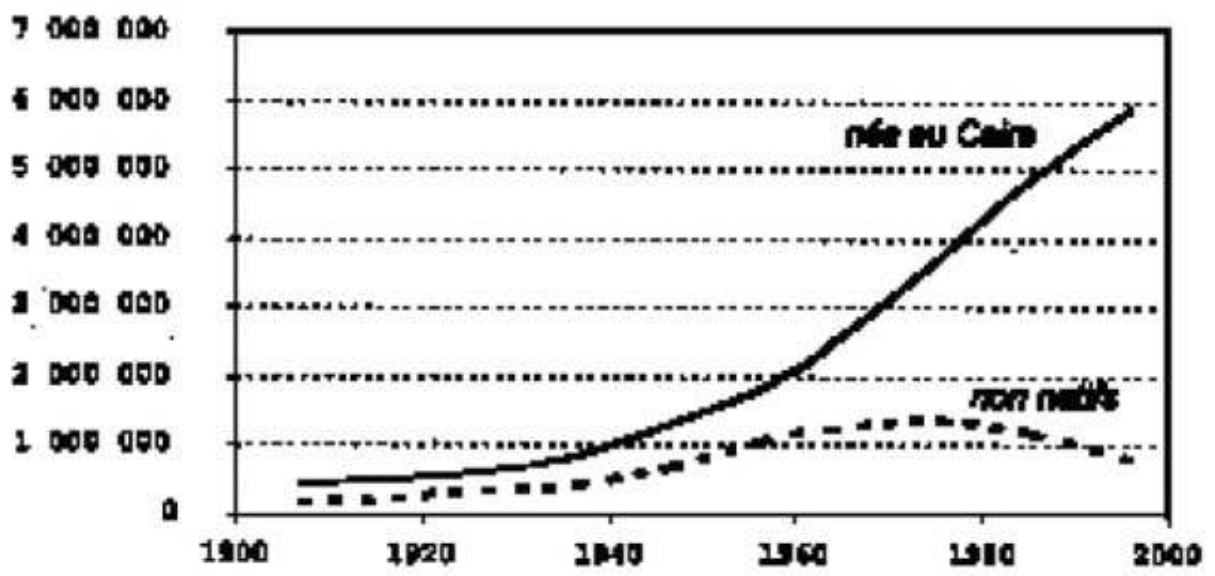

Sources : recensements égyptiens.

Dans les années 1970, la capitale est très largement sous-équipée. La pression accrue sur des infrastructures cairotes vieillissantes renforce l'impression de submersion. Les égouts débordent, les logements précaires sur les toits ou dans les cimetières se multiplient et les bus, avec des passagers accrochés jusqu'à l'extérieur des véhicules, deviennent une image caractéristique du quotidien cairote (Waterburry, 1973). Dans un contexte de pénurie financière, de conflits et d'efforts militaires, les politiques urbaines disposent de peu de moyens. L'extension des réseaux et leur modernisation sont sans cesse retardées au profit des seuls chantiers d'urgence. De même, les villes de province n'ont fait l'objet d'aucun effort notable. Elles ont même souffert de la concentration extrême de l'effort industriel autour de la capitale, surtout celles du sud de l'Égypte qui ont reçu à peine $5 \%$ des investissements.

La paix retrouvée, malgré un budget de l'État en forte croissance avec l'essor des rentes et des taxes d'importation, les chantiers indispensables à la modernisation de la capitale continuent d'absorber l'essentiel des finances disponibles. En 1976, le budget d'investissement destiné à la région métropolitaine représente 37,5\% des capacités d'investissement public ${ }^{11}$. De même, les importantes coopérations internationales et bilatérales qui suivent les accords de Camp David privilégient la mise à niveau des équipements cairotes. Depuis plus de 20 ans, Le Caire est le théâtre d'énormes chantiers d'infrastructures, que ce soit pour la desserte en eau potable, le drainage sanitaire, dont le réseau remonte au début du siècle, et l'accessibilité, avec les voies rapides aériennes, le boulevard périphérique et ses ponts sur le Nil ou les deux lignes de métro.

La contrainte métropolitaine réside dans cet impératif besoin de garantir le fonctionnement de la capitale où tout est concentré. Surtout, l'accumulation des pouvoirs doit cohabiter avec une foule urbaine qu'il faut bien satisfaire, sous peine de voir le système menacé par l'émeute. Qu'une ville de province reste totalement souséquipée, et le pouvoir ne sera pas immédiatement contesté, l'intervention policière suffira à maintenir l'ordre. Mais qu'au Caire, le peuple arrive à exprimer son mécontentement dans la rue, à déborder l'îlotage policier et c'est le pouvoir central, directement, qui se voit en péril. L'agitation de ce spectre induit très largement l'orientation des politiques urbaines, de la non-démocratisation aux subventions alimentaires. En 1977, le gouvernement Sâdât [Sadate] sait bien jouer de cette co- 
présence du pouvoir et du peuple comme risque politique majeur. Il laisse monter la contestation jusqu'à l'émeute, au moment où il négocie sa politique d'endettement et de dépenses auprès du Fonds monétaire international (Sadowski, 1991). Les grands chantiers d'infrastructures suivront et les subventions aux produits de base ne seront plus guère contestées; celle du pain en particulier, qui assure encore aujourd'hui $60 \%$ de l'apport calorique quotidien en ville. La nécessité d'équiper, de suréquiper au regard des besoins des provinces, s'explique, en dehors de ces impératifs de maintien de l'ordre, par le simple appel cumulatif à la modernisation qu'impose la concentration de l'appareil économique au Caire.

Débordements, dessertes et mises à niveau, ou la naissance du biais métropolitain

La modernisation des infrastructures est contrariée par un puissant mouvement d'expansion vertical et horizontal du cadre bâti. Entre 1972 et 1978, l'agglomération métropolitaine s'étend de plus de 3,5\% par an, puis de 1,5\% de 1978 à 1986 et encore de $1 \%$ jusqu'en 1994 (tab. 1). Une dynamique de construction davantage portée par le secteur privé prend le relais, élargit et étend les couloirs d'urbanisation ouverts par la politique d'industrialisation des années 1960 . Les taux de croissance du nombre de logements, plus importants que ceux de l'aire agglomérée, reflètent la forme urbaine dominante qui s'impose à partir des années 1970 : l'étalement est minimum et la densification, par bourrage et verticalisation du bâti, devient maximale. Les villes de province connaîtront à leur échelle une évolution semblable.

Tableau 1 : Dynamique de la construction au Caire 1950-2000 (taux annuel de croissance)

\begin{tabular}{|l|l|l|l|l|l|l|}
\hline \multicolumn{2}{|c|}{ Taux annuel } & $90 / 96$ & $80 / 89$ & $70 / 79$ & $60 / 69$ & $50 / 59$ \\
\hline \hline Grand-Caire & Population & 2,0 & 2,8 & 2,7 & 3,9 & 3,8 \\
\hline \hline & Logements & 3,5 & 4,6 & 4,8 & 5,1 & 1,7 \\
\hline \hline Le Caire & Logements & 3,0 & 3,7 & 4,0 & 4,4 & 1,9 \\
\hline \hline Qalyûbiyya & Logements & 4,2 & 5,4 & 6,3 & 7,7 & 1,1 \\
\hline \hline Giza & Logements & 3,7 & 5,3 & 5,9 & 5,9 & 1,8 \\
\hline
\end{tabular}

Source : recensement de l'habitat 1996 - BDG EGIPTE-CEDEJ

Durant la période 1950-1959, la croissance de la population est plus forte que celle du logement. C'est alors qu'apparaissent les poches d'habitat précaire, les logements sur les toits et dans les cimetières. Ensuite, la croissance économique stimule un marché immobilier adapté à la demande populaire : l'informel (El Kadi, 1988). Le défi de la croissance mégapolitaine s'impose selon ces termes jusqu'à nos jours. Sur la base d'une appréciation tardive de la poussée démographique, les efforts d'équipement restent toujours en retard par rapport à la dynamique de production de la ville. L'impression d'explosion urbaine est le produit de cette double dynamique des chantiers permanents liés, d'une part, aux besoins d'infrastructures et, d'autre part, au boum de la construction. 
39 Face à la crise grave des infrastructures engendrée par l'absence de prévision des conséquences de la croissance démographique et, surtout, le manque de moyens d'intervention, les gouvernements successifs se sont rendus à l'analyse la plus simple, qui consiste, en cœur avec les citadins, à stigmatiser les migrants comme responsables de la saturation des infrastructures, comme " ruralisant » la ville et la dégradant. Cette politique du verbe borne la sphère citoyenne, donne en pâture des boucs émissaires et voile, au moindre coût, la grande faiblesse des moyens dégagés pour mettre en œuvre une politique urbaine. Ce bornage du monde citadin légitime, instauré en mode de gouvernement, a eu aussi pour effet de renforcer le caractère marginal et illégal de la filière de promotions immobilières populaires. Il a légitimé son exclusion des services urbains. Ce sont des choix qui, à présent, pèsent lourdement sur les finances publiques et imposent des efforts de rattrapage considérables, mais devenus inévitables tant la filière illégale est désormais majoritaire et fait partie de la métropole. Mais pour l'essentiel, les habitants ont financé les services, y compris des réseaux scolaires privés, et ils continuent, selon la logique de l'ajustement, du "qui consomme, paie » - une condition désormais érigée en principe universel d'accès à la ville, au statut de sociétaire des services urbains.

Si la production de logements explose dans les années 1960-1970 et reste jusqu'à aujourd'hui très nettement supérieure à la croissance de la population, c'est bien sûr parce qu'il existe une demande, notamment une demande populaire, mais c'est aussi, voire surtout, parce que, jusqu'à une période très récente, l'inflation était très élevée : elle dépassait $10 \%$ par an. La construction et le placement immobilier ont donc été les moyens les plus sûrs de ne pas perdre des gains obtenus dans le commerce, l'industrie ou lors d'une migration. Les remises des émigrés dans les monarchies pétrolières ont ainsi constitué la base essentielle de la dynamique immobilière dans tous les segments du marché jusqu'à une période très récente. Répondant à une logique de placement, l'effort de construction ne rencontre pas forcément la demande. Ceci explique en partie l'existence d'un million de logements vides au Caire en 1996 et de deux millions pour toute l'Égypte, alors que la part de logements insalubres, d'une seule pièce ou partagés par plusieurs familles reste considérable. Les législations successives sur le blocage des loyers et l'absence d'accès au crédit sont d'autres éléments explicatifs qui mériteraient en eux-mêmes des développements. Soulignons simplement que l'absence ou plutôt le laminage de la classe moyenne, depuis la fin des années 1960, a largement contribué à produire cette forme urbaine duale. D'une part, un segment réduit de citadins enrichis par la dérégulation et les affaires placent leurs plus-values dans l'immobilier de standing et, d'autre part, une masse de familles n'ayant pas accès aux crédits bancaires se tournent vers le marché populaire illégal de production de logements. La faiblesse des salaires et l'instabilité professionnelle sont des facteurs surdéterminant la production des paysages urbains contemporains. Ce modèle s'oppose à l'association banque publique - fonctionnaires - emplois à vie - classe moyenne qui a produit l'univers urbain européen contemporain. L'irrégularité ou l'insécurité professionnelle associée à la faiblesse des revenus donne des paysages urbains spécifiques marqués, notamment, par l'hétérogénéité, les friches, la dégradation ou l'inachevé... Par l'instabilité.

41 Désormais, avec la maîtrise de l'inflation, très nettement en dessous de $10 \%$ par an depuis près de 10 ans, les petits transports privés, les taxis et minibus, voire la petite manufacture, captent une part sans cesse croissante des remises des travailleurs à 
l'étranger - sachant que le nombre de ces derniers, de plus en plus en concurrence avec la main-d'œuvre qualifiée asiatique, tend à se réduire. Toutefois, près de deux millions d'Égyptiens travaillent toujours à l'étranger, essentiellement dans les pays du Golfe, et leurs remises sont encore comparables aux revenus du tourisme.

Le redéploiement post-1952La transition : de l'espace-distance des villes à l'espacetemps de l'urbain

devenant une forme très populaire d'entreprenariat, qui dépasse désormais la captation de la seule épargne des immigrés dans les monarchies pétrolières, l'investissement dans les transports publics intra et inter-urbains, encore facilité par les formules désormais courantes de location-vente, participe de l'équi-habitabilité de l'espace égyptien, autrement dit: de l'équivalence de la localisation du lieu de résidence. Il a permis d'accélérer, dans l'actualité des dix dernières années, la tendance amorcée, dès la fin des années 1960, à la réduction des migrations définitives au profit des migrations pendulaires.

Avec la métropole comme extension définitive et intégratrice de l'ensemble du territoire, ce n'est plus la distance qui compte mais l'accessibilité, le temps de parcours. Nous sommes donc passés d'un monde de villes organisatrices d'un espace-distance à un univers urbain reposant sur la maitrise croissante de l'espace-temps. On passe ainsi d'une structure de peuplement modelée par des temporalités longues induisant des mouvements, notamment migratoires, irréversibles, à un territoire re-configuré par des temporalités courtes et réversibles, se caractérisant aussi par la multiplication des ancrages ou des appartenances. Dans le même temps, alors que le rapport des individus à l'espace devient multiple, le mode de peuplement du territoire s'unifie et s'homogénéise. Ainsi se dessine la chaîne génératrice de l'univers métropolisé contemporain avec, d'abord, les villes, puis les auréoles urbaines qui débordent l'administration des villes et, enfin, la métropole qui fédère, polarise et redistribue en imposant son gouvernement du système peuplement.

La chronologie nous ramène au basculement politique des années 1960. Vu du Caire, la période se caractérise, nous l'avons dit, par le fléchissement de la croissance démographique et son caractère de plus en plus endogène, qui s'accompagne d'une transformation des milieux locaux, avec des villages dont les activités se diversifient.

La politique de redistribution des terres, mais surtout les bonifications et les grands chantiers réduisent l'exode rural. Bientôt, les flux migratoires dominants s'opèrent, d'une part, entre villes et, d'autre part, entre villages, voire encore, s'orientent de l'urbain vers les secteurs ruraux. Surtout, les distances parcourues sont de plus en plus faibles. Parallèlement, les migrations des campagnes vers les villes n'ont cessé de fléchir jusqu'à leur quasi-épuisement (8 \% du total des migrations en 1996).

Le bornage et l'élargissement du territoire utile, entreprise marquante de l'effort d'affirmation du nouveau régime, avec des chantiers tels que le barrage d'Assouan ou la conquête de nouvelles terres et la fondation de villages modèles, comme en Buhayra, contribuent au redéploiement centrifuge de la dynamique de peuplement et impulsent la micro-urbanisation diffuse.

Le Haut-Barrage n'est pas seulement un facteur conjoncturel de migration vers le sud, mais un puissant moteur de déconcentration. L'entreprise écarte les risques de sécheresse ${ }^{12}$ et met hors d'eau la section de la Haute-Vallée du Nil encore soumise à la crue. Il achève ainsi l'entreprise de passage à l'irrigation pérenne commencée sous le 
règne de Muhammad ${ }^{\mathrm{A}} \mathrm{Ali}$. Ce chantier séculaire de maîtrise du cours Nil, d'extension du réseau des canaux et des drains, destiné à accroître les rendements et les surfaces agricoles, a aussi favorisé l'étalement du peuplement urbain au détriment de l'agriculture. En effet, lorsque le régime de la crue disparaît, les villages et les villes peuvent descendre du relief - du Kûm - ou des escarpements en bordure de la Vallée, qui les protègent de l'inondation. C'est le point de départ de l'urbanisation sur place, in situ.

De plus, le Haut-Barrage, en réduisant le débit du Nil, dégage de nouvelles terres sur les berges jusqu'alors inondables, offrant ainsi des opportunités étendues à la filière foncière agricole et à l'urbanisation des villages. Il introduit ainsi une nouvelle richesse dans de nombreux villages. Enfin, le Haut-Barrage est d'autant plus propice à la croissance sur place qu'il apporte aussi une énergie électrique abondante, favorable au développement des activités manufacturières tant à grande échelle, avec la sidérurgie à Assouan ou Nagc Hammâdî, qu'en favorisant l'essaimage de micro-ateliers dans les bourgs et les villages.

Les effets des invasions-destructions et de la guerre d'usure de 1956 à 1973 sur le peuplement du canal de Suez brouille de façon simplement conjoncturelle l'inéluctable tendance à la déconcentration. Les réfugiés du Canal, s'ils ont largement contribué à l'encombrement et à la saturation si prégnante des années 1960, tant au Caire qu'à Alexandrie, rejoindront, pour la plupart, avec de nouveaux migrants, les villes du Canal après 1973. Beaucoup encore, temporairement, retrouveront leur village ou ville d'origine.

Après 1952, la réorganisation du système de production agricole s'accompagne d'un indiscutable effort de modernisation, notamment des transports, en même temps que des autres réseaux, de l'électricité en particulier, qui favorise le désenclavement et l'intégration du territoire. La réforme agraire, la redistribution des terres en particulier, a permis que l'intensification des rendements ne se traduise pas immédiatement par une nouvelle réduction des besoins en main-d'œuvre de l'agriculture. Elle a servi de tampon jusqu'à ce qu'il se crée, dans les bourgs et les villages, d'abord des emplois de fonctionnaires, d'encadrement, puis une demande locale croissante pour des services privés, notamment en transport et en accès à des biens de consommation courants. Il a ainsi été créé un climat favorable à l'épuisement de l'exode rural, malgré l'accumulation, au Caire en particulier, de l'investissement et une évidente captation des revenus de la terre en faveur de l'effort de guerre et d'industrialisation.

51 L'accélération de la tendance centrifuge a dû attendre la convergence de deux transformations : d'une part, la réduction de l'espace-temps, avec la modernisation des transports et surtout leur plus grande flexibilité liée à l'explosion du parc des microbus ; et, d'autre part, le passage progressif, à partir des années 1970, d'une économie de pénurie à une économie de consommation, qui favorise la disponibilité et l'accessibilité des biens et des services et donc la déconcentration de la croissance. La flexibilité accrue des transports, le passage du train à la route avec, en particulier, l'explosion du nombre de micro-bus, réduit les effets de corridor et permet une dispersion de l'activité au-delà des grands axes desservis par le chemin de fer, en dehors des métropoles régionales et de leurs périphéries immédiates.

L'aboutissement : de la micro-urbanisation à la coalescence 
Ainsi est né et s'est affirmé un paysage mité de villages en voie d'urbanisation et de bourgs qui prennent de plus en plus des allures de ville, tant par les services qu'ils offrent que par leur morphologie ou les modes de vie qui s'y transforment (Fanchette, 1997). Si, de façon conventionnelle, on se fixe la limite de 10000 habitants agglomérés dans une même unité morphologique comme définition minimum de ce qui est urbain, en dehors de toute considération administrative, la nuée issue de la déconcentration et de l'étalement apparaît clairement. La micro-urbanisation devient même la catégorie de peuplement la plus caractéristique de l'Égypte (tab. 2). C'est, en tous cas, celle qui connaît la plus forte croissance (graph. 3).

Tableau 2 : Distribution de la population par type d'agglomération morphologique (\%)

\begin{tabular}{|l|l|l|l|l|l|l|l|l|}
\hline Type d'agglomération & 1947 & 1960 & 1966 & 1976 & 1986 & 1996 & 2010 \\
\hline \hline Agglomération urbaine & 25,9 & 31,8 & 34,3 & 36,4 & 38,4 & 37,2 & 34,1 \\
\hline \hline Villes & 4,4 & 4,6 & 5,1 & 5,6 & 5,9 & 7,1 & 6,7 \\
\hline \hline Agglomération de villages & 2,7 & 2,9 & 3,2 & 3,5 & 5,7 & 6,2 & 8,8 \\
\hline \hline Villages urbains > 10000 Habts & 3,2 & 4,6 & 5,7 & 8,0 & 12,5 & 15,8 & 20,1 \\
\hline \hline Population urbaine & 36,2 & 43,9 & 48,3 & 53,5 & 62,5 & 66,2 & 69,7 \\
\hline \hline Villages < 10000 ou rural dispersé & 63,8 & 56,1 & 51,7 & 46,5 & 37,5 & 33,8 & 32,1 \\
\hline
\end{tabular}

Source : recensements de la population, BDG EGIPTE-CEDEJ et projection, hypothèse basse (É. Denis).

Le paysage est évidemment en mouvement. Ce que nous observons ici est transitoire. Se dessine en effet une propension à la coalescence de plus en plus généralisée du système de peuplement. L'étroitesse de l'espace habité et les couloirs d'urbanisation anciens, en particulier les berges du Nil et les canaux primaires accueillant aussi les voies de circulation majeures, favorisent cette tendance.

Le critère de 10000 habitants, tout conventionnel qu'il soit, correspond aussi à un seuil d'équipement et de basculement des besoins d'organisation liés à la proximité et à l'organisation de la communauté. Des services collectifs deviennent nécessaires, des réseaux s'imposent - voiries, eaux, égouts... L'agglomération stimule aussi la diversification et la multiplication des compétences, l'offre de services privés peut s'épanouir. Autour des stations de micro-bus « spontanées » qui s'implantent à la sortie du moindre village, fleurissent peu à peu des cafés, des garages, puis des restaurants et des épiceries... La morphologie des villages elle-même se modifie ; ces plates-formes de transport deviennent des péri-centres qui captent l'activité locale et la détournent des centres anciens désormais enclavés.

Selon ce critère morphologique, l'Égypte compte plus de 800 agglomérations de plus de 10000 habitants contre seulement 150 villes officielles. Le taux d'urbanisation ainsi obtenu, 67 \% en 1996, soit $24 \%$ de plus que le taux officiel, correspond aussi aux taux cumulés d'actifs des secteurs secondaires et tertiaires. Il est donc représentatif d'une 
morphologie et d'une géographie du peuplement en accord avec les transformations socio-économiques.

Graphique 3 : Répartition cumulée de la population par taille d'agglomération morphologique en 1996

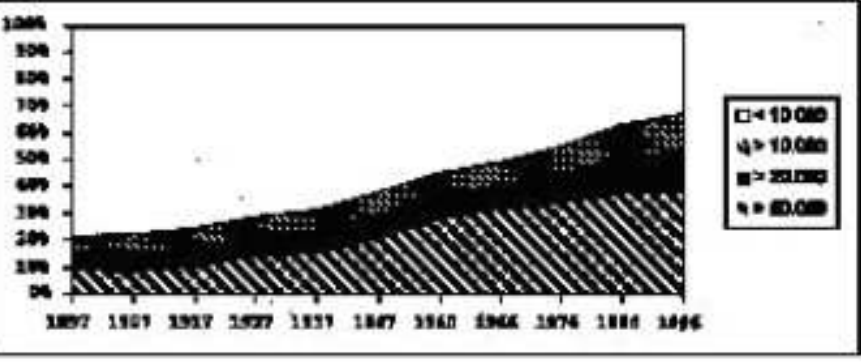

Source : recensements de la population, BDG EGITE-CEDEJ

Parce que, jusqu'à preuve du contraire, l'écoumène égyptien est un espace fini, l'aboutissement de la dispersion du peuplement s'apparente à l'homogénéisation ou à la convergence placée sous le signe de l'urbain, avec une agriculture intégrée et interstitielle relevant du jardinage, pour le moins du maraîchage à forte valeur ajoutée, à l'image de ce que l'on peut observer dans le delta hollandais (cf. tab. 3) ou dans les plaines côtières japonaises. La dispersion jusqu'à la coalescence, ainsi nous retrouvons l'« écouménopolis ».

Tableau 3 : Comparaison Égypte et Pays-Bas

\begin{tabular}{|c|c|c|}
\hline & Égypte & Pays-Bas \\
\hline Part de l'agriculture dans le PIB en 1996 & $17,0 \%$ & $6,4 \%$ \\
\hline Valeur de l'agriculture en US\$ en 1996 & 10712150000 & 14290141500 \\
\hline Surface cultivée en km2 & 26070 & 9300 \\
\hline Productivité par km2 cultivé en US\$ & 410900 & 1536574 \\
\hline Productivité métropolitaine par $\mathrm{km}^{2}$ en US\$* & 33117647 & 45755690 \\
\hline Surface habitée en $\mathrm{km} 2$ & 35000 & 33000 \\
\hline Densité en 1996 par km2 & 1714 & 455 \\
\hline Population totale en 1996 & 60000000 & 15000000 \\
\hline Population active agricole en 1996 & 4000000 & 120000 \\
\hline Habitants par cultivateur & 15 & 125 \\
\hline Productivité par agriculteur en US\$ & 2678 & 119085 \\
\hline
\end{tabular}




\begin{tabular}{|l|l|l|}
\hline Population totale en 1882 & 4600000 & 6700000 \\
\hline \hline Densité en 1882 par km2** & 139 & 223 \\
\hline
\end{tabular}

Sources : recensements, BDG Géopolis (F. Moriconi), rapports annuels, FAO.

* Les productivités urbaines par $\mathrm{km}^{2}$ sont assez proches du fait de l'extrême densité de la métropole égyptienne. L'écart entre urbain et rural (rural réduit à l'agricole) est, en revanche, beaucoup plus fort en Égypte qu'aux Pays-Bas, signe d'un déficit d'intégration de la filière agricole et de l'extrême polarisation de l'espace productif égyptien.

** Depuis 1882, les Pays-Bas utiles se sont étendus d'environ 2000 km², par poldérisation, et l'Égypte utile de $3000 \mathrm{~km}^{2}$, par drainage et bonification.

L'humanisation extrême du territoire combinée à l'accessibilité conduit à une équihabitabilité des lieux. Telle est la tendance, mais elle est profondément complexifiée par les ressources locales et les traces matérielles qui composent, à travers la manière dont elles sont mobilisées, la diversité et la disparité des topographies locales. Elle est surtout extrêmement contrariée par un centralisme autoritaire qui ne fléchit pas, envisageant la libéralisation sous le seul aspect économique avec une désétatisation comprise comme le simple synonyme du transfert des monopoles du public au privé, mais sans remise en cause de l'imbrication des intérêts de l'entreprise et du politique de l'affairisme.

Alors que le temps des villes était, nous l'avons dit, porteur d'une pluralité de gouvernements, d'un municipalisme émergeant et donc d'une émancipation des villes, la métropolisation s'accompagne, en Égypte - mais il ne s'agit en aucun cas, ici, d'ériger l'Égypte en exception -, d'une forme de plus en plus impériale et autoritaire, sans partage ni dialogue, d'administrer le territoire. Rappelons simplement qu'en 1995, l'élection des maires a été supprimée, sous couvert de contrôle de la poussée islamiste, ce qui répondait certes à des impératifs de politique nationale, mais n'était pas sans conséquences sur la conduite des affaires locales. En ce sens, l'islamisme apparaît, dans le jeu des représentations qui président à la maîtrise du territoire, comme le contrepoint nécessaire à la pacification et à l'unification de l'imperium. J'utilise à dessein l'idée d'imperium pour souligner le caractère de plus en plus ressemblant du modèle métropolitain de type égyptien avec la forme classique de la cité-état - nous y reviendrons.

Gouverner ou diriger la ville égyptienneDéveloppement à la marge et convergence ville-campagne

La formation de ce vaste ensemble archipélagique lie encore davantage ville et campagne et brouille définitivement les limites non seulement morphologiques mais aussi sociales.

Depuis les années 1970-1980, la croissance démographique urbaine n'est plus absorbée par les tissus existants mais génère un étalement sur les franges agricoles. C'est une rupture fondamentale dans la production des villes et le passage du monde des villes aux territoires urbains. Elle est encore plus liée à la libéralisation économique qu'on ne le pense. Ce n'est pas seulement une question liée à l'investissement des remises des travailleurs immigrés, à l'inflation ou à la disponibilité des matériaux de construction avec l'industrialisation, c'est avant tout une question de changement des rapports au foncier agricole. La dérégulation de l'agriculture a rendu possible la transaction du sol et le transfert des usages agricoles aux usages urbains. Ceci n'est réalisable que parce 
que, préalablement, la réforme agraire a permis une redistribution des terres et donc une réappropriation des valeurs foncières. En ce sens, la question urbaine est totalement interdépendante de la question agraire et de leur régulation croissante par les seules "forces» du marché. Le renforcement des dispositifs légaux supposés contraindre l'étalement urbain est, de ce point de vue, en parfaite contradiction avec la logique libérale qui prévaut à présent, et la réponse massive par l'illégalité de la subdivision du sol et de la construction est une manière de manifester sa volonté d'intégration à la logique dominante de production de la ville. Les marges informelles représentent le produit le plus répandu et le plus caractéristique de la libéralisation, celui qui loge et procure une activité à la majorité.

Ignorant ou plutôt masquant ces transformations, l'un des efforts majeurs de catégorisation politique et médiatique correspondant à la phase de libéralisation économique active de ces dix dernières années a pour objet de typifier les habitants des quartiers paralégaux périphériques en "autres intérieurs». Cette représentation dominante du monde social et le bornage de ses territoires ainsi produit contribuent à préserver l'intangibilité des frontières de ce qui est légitimement citadin dans un contexte d'intense étalement remettant précisément en cause l'existence de ses limites. Elle a aussi pour objet de localiser un bouc émissaire, un responsable de la dégradation des conditions de vie urbaine. Les habitants des quartiers paralégaux où cashwâ'iyyîn ont remplacé dans l'imaginaire politique les fallâhîn qui envahissaient les villes et la «ruralisaient». La représentation active enregistre donc le changement de configuration et la quasi-disparition des migrations du monde rural vers les villes. Désormais, le mal est à l'intérieur. Il ceinture et dénature la cité. Ce refus de la banalisation du processus spontané de déconcentration et d'étalement renvoie à la volonté de ne pas partager le pouvoir politique et de ne pas décentraliser. Il s'agit par là-même de survaloriser les citadins de souche, de réifier un type cairote légitime immuable, afin de valider une politique hégémonique métropolitaine, fondée sur le drainage des richesses vers la capitale et la colonisation des provinces. Pour le moins, les catégories sont-elles figées et l'intégration urbaine bloquée, contrariée en dehors de l'équipement en services, qui progresse uniquement en raison de contraintes techniques, parce que les réseaux imposent l'interdépendance des lieux habités. Mais cela ne va guère plus loin. Simplement, des interventions policières de reconquête territoriale d'esprit purement colonial concernant des quartiers dits informels, comme à Imbâba en 1994 (Denis, 1995), contiennent aussi l'ambition de transformer les habitants, de les éduquer, tout en leur déniant des compétences, dans le plus pur esprit de la réforme sociale, et en leur refusant forcément toute marge d'autonomie de gestion, de représentation ou de participation politique.

L'écologie et la citoyenneté privée

La transfiguration de la représentation du Caire et, par extension, des problèmes urbains en Égypte, polarisée désormais par la question des quartiers cashwầ, s'accompagne d'une affirmation des thèmes écologiques. La pollution est devenue un thème central, renvoyant certes à une réalité très prégnante, mais qui a aussi pour effet d'ouvrir la voie à l'assimilation entre la pollution et la dégradation de la qualité de la vie du fait de l'informalité et de ses habitants, par nature sales et ne pouvant produire qu'un environnement dégradé. Ce discours est formulé comme l'acte de décès nostalgique d'une vie citadine idéalisée: la ville est désormais totalement et radicalement corrompue, perdue. Il marque en ce sens une rupture avec les représentations réformistes antérieures, qui avaient encore pour objet de maitriser la 
ville. C'était très clair avec le boulevard périphérique, dernière enceinte pour protéger l'intégrité de la cité. Désormais, elle est déclarée totalement dégradée.

Ce basculement sert d'appui à deux formes de redéfinition et d'intervention sur le territoire urbain. D'une part, il plaide en faveur d'opérations dures d'expulsion et de reconquête territoriale, de destruction de logements insalubres au profit d'espaces verts, ainsi qu'à l'ambition de se réapproprier une vieille ville dégradée, le centre ancien dit «islamique » ou "fatimide », pour en faire un musée à ciel ouvert, un parc d'attraction débarrassé de ses activités artisanales et de sa population vieillissante et déshéritée. D'autre part, il a servi de base argumentaire pour la promotion de nouvelles formes d'habiter pour les plus riches, de résidences fermées et gardées sur les marges désertiques ou gated communities. Le boum immobilier de ce produit exclusif qui s'accompagne de parcs d'attraction et de golfs, pur résultat de la libéralisation et de la dérégulation, a eu pour effet morphologique, depuis moins de dix ans, de doubler la surface bâtie ou en construction dans l'agglomération du Grand-Caire. Il juxtapose aussi un tissu très lâche, une urbanité radicalement différente, une nouvelle manière de concevoir la ville, à l'une des métropoles les plus denses du monde avec Bombay. Ce produit immobilier invitant à un mode de vie radicalement nouveau en Égypte a été extrêmement bien accueilli par les élites et classes moyennes supérieures qui ont massivement acheté. Il matérialise un véritable désir de distinction qui ne peut s'exprimer qu'en dehors de la ville considérée à présent comme trop dense, polluée, appauvrie et trop mixte socialement - autant de caractéristiques pas si nouvelles. Il s'agit de pouvoir habiter entre soi et sans attendre une croissance plus partagée. La formule va jusqu'à proposer, à l'échelle de la communauté enclose, des élections et une communauté de gestion; comme si, aujourd'hui, la seule démocratie possible était privée et en marge de la sphère publique. Il y a clairement une volonté de désengagement, pour le moins, de moindre interaction avec des non-semblables et de vie loin de la moralisation de l'espace public. La privatisation passe par exemple, au moment de l'achat d'une résidence, par la souscription à un fonds commun de placement en bourse dont les revenus doivent rapporter les sommes nécessaires à l'entretien des parties communes et au gardiennage.

L'« écouménopolis » contrarié

64 Le processus morphologique, la convergence des formes de peuplement de la Vallée et du Delta du Nil, ne dit rien du mode de gouvernement et de la hiérarchisation de la formation socio-spatiale en construction. Il inviterait à penser qu'inévitablement, suivant le paradigme de la modernisation et les principes généraux de sens et de finalités de l'histoire, qu'une décentralisation politique prolongera la déconcentration démographique. Mais le centralisme politique autoritaire et la polarisation économique invitent à envisager un scénario radicalement opposé, de même que ces nouvelles formes résidentielles des élites métropolitaines. Il n'est d'ailleurs pas certain, compte tenu de l'intensité de la métropolisation économique, que la stabilisation démographique et territoriale soit acquise durablement. Il est possible, en effet, qu'elle préfigure dans ses excès un nouveau cycle de migration vers la métropole et ses villes nouvelles industrielles, où campe déjà une main-d'œuvre flottante en attente d'embauche journalière.

Si les familles égyptiennes, en excluant le changement de résidence, ont pour stratégie de maximiser les réseaux de proximité, comme la famille et l'inter-connaissance de la communauté, renforçant ainsi la cohérence des tissus locaux, le gouvernement de 
l'Égypte a toujours pour objet un strict encadrement hiérarchique qui laisse peu de place à la représentation active et encore moins à la réforme du maillage administratif et politique.

Le renforcement des localités accompagne la quasi-résorption de l'hémorragie des forces vives et des compétences qui contrariaient leur potentiel de diversification et d'essor jusqu'aux années 1960-1970. La révolution des transports, les migrations pendulaires, l'accès aux services et aux marchandises, y compris aux biens d'équipement, aux machines pour les activités manufacturières ou tout simplement aux frigos pour les restaurants, ont impulsé l'émergence d'une nouvelle localité élargie et complexifiée. Or, elle n'est pas transformée simplement du point de vue économique, mais elle l'est aussi socialement, voire politiquement et culturellement. La microurbanisation est, en effet, le produit de l'activation ou de la réactivation des ressources locales, des compétences et du capital disponibles, pour exploiter les potentialités d'une insertion nouvelle dans la trame égyptienne et plus largement l'ouverture et l'accès au monde. La compression de l'espace-temps, des distances, est évidemment le vecteur fondamental de cette recomposition. C'est cette combinaison de relocalisation et d'ouverture qui forme la spécificité de ce qui est en jeu. Dans cette géographie, il n'y pas de contradiction entre tradition et modernité. Des institutions localisées, comme la famille, voire l'appartenance tribale, en pleine réactivation et réécriture dans le Sa'îd (ou Sud égyptien), servent d'appui à la négociation de la modernisation de la localité.

Dans le contexte antérieur, fait de migration vers les métropoles, la modernisation supposait la rupture avec le capital familial et un certain effacement des appartenances régionales. Au mieux, les ligues locales et régionales servaient de point d'insertion dans l'apprentissage de la ville. Le capital familial et culturel prend un sens tout à fait différent dans le contexte actuel d'accessibilité extrême, différent des phases antérieures, quand la pénurie imposait la migration définitive. Tout en se réinventant, la localité d'appartenance devient un capital. Ces expressions situées sont aussi des contre-modèles qui servent à légitimer l'autoritarisme du système dominant. Elles sont, parfois encore, phagocytées à travers un travail de folklorisation-stigmatisation, notamment dans le cas du Sacîd, comme le montrerait la trajectoire du poète exmilitant 'Abd al-Rahmân al-Abnûdî ou, plus encore, le travail médiatique autour de la Nubie, de plus en plus comprise comme une africanité intérieure. Ainsi s'entretient aussi une culture dominante stigmatisant les différences et l'arriération de tout ce qui n'est pas métropolitain.

Cent ans de métropolisation : d'un impérialisme à l'autre

La vague séculaire d'agglomération des activités et des populations a transformé l'Égypte des villes en un monde urbain apparemment uniformisé, mais, en fait, encore plus polarisé qu'auparavant par sa capitale, par le Caire.

Malgré le tassement précoce de l'exode rural, l'aspiration métropolitaine a été renforcée par le projet d'édification nationale après 1952. Sa mise en oeuvre a ouvert la voie à une polarisation de l'effort d'investissement qui, lorsque le projet de construction nationale a perdu ses ambitions de libération pour se crisper sur le nationalisme, s'est révélé sans fard au service d'une machine de sujétion organisant l'exploitation du territoire utile et la conquête de nouveaux espaces, de l'arrière-pays ou du pays arriéré. Ainsi, à l'édification inachevée de l'État-nation s'est substitué un projet de métropole-État que masque de plus en plus mal un travail d'activation de l'identité nationale manquant de légitimité, voire de projet crédible. Plus exactement, 
le modèle classique de la cité-État est le mieux à même de schématiser la configuration territoriale qui organise la société égyptienne aujourd'hui et son mode de gouvernement. Comme si les principes de la cité-État étaient de ceux qui décrivaient le plus « objectivement» le rapport d'un gouvernement et d'une société à son territoire, de ceux qui laissent le moins de place pour les représentations totalisantes de type nationaliste et oblitèrent le moins le caractère impérialiste des systèmes de peuplement et leur tendance à l'accumulation métropolitaine. Sans doute aussi que le caractère insulaire de l'Égypte habitée invite à ce parallèle anachronique.

Sans redéployer notre argumentaire, remarquons simplement qu'au Caire, aujourd'hui, comme dans de nombreuses métropoles du Sud et en Grèce antique, la citoyenneté est dépendante du droit de propriété. Il existe une catégorie d'habitants des faubourgs non citadins, sans droits, qui vivent dans l'illégalité foncière et immobilière. Ce mode de gouvernement postule le silence des non-citoyens, leur absence de voix, alors que la représentation active est réservée à une oligarchie d'entrepreneurs fortunés qui maintiennent le système politique aux marges de la pure tyrannie.

71 Selon cette même perspective, les politiques de grands travaux, de "mégaprojets ", pour reprendre la terminologie officielle, comme la mise en culture irriguée du 2300 $\mathrm{km}^{2}$ de désert à l'ouest du lac Nasser lancée en 1997, à Tushkâ, sont une forme d'extension de l'imperium, de conquête et d'exploitation minière des ressources comme d'occupation du vide. Ces objectifs de colonisation intérieure sont aussi visibles et crédibles que le discours qui consiste à les présenter comme des projets d'intérêt national. Le méga-projet représente une forme aboutie de réconciliation entre la mobilisation de la communauté nationale et l'ambition impériale. Elle est certes en contradiction avec le libéralisme et la désétatisation, mais elle constitue aussi une passerelle entre l'exploitation du territoire et la métropolisation; elle tend à réconcilier les contradictions géographiques - l'étendue et le pôle ou le dominé et le dominant. C'est dans cette tension que se dynamisent les géographies de notre monde, cet archipel de mégapoles qui, à toutes les échelles, maitrisent les circulations et contrôlent ainsi les étendues, les économies, les idées et les peuples.

Le siècle, encore, peut être compris comme la transfiguration de l'imperium: il se contracte, change d'échelle avec le retrait des Britanniques et la désaffiliation de leur empire, puis les impératifs de la construction nationale oblitèrent la dimension impériale mais sans la remettre radicalement en cause et, enfin, avec la libéralisation économique, la configuration impériale et coloniale polarisée par la métropole est redynamisée. Nous retiendrons que l'Égypte est en voie de métropolisation totale. Loin donc du modèle théorique de "l'écouménopolis", où une multiplicité d'échanges entretenant la diversité se développent sans l'intermédiaire du gouvernement central.

BIBLIOGRAPHIE

Akouri M.,1993, « Chronique culturelle », Égypte-Monde Arabe, n 14. 
Alleaume G. \& Denis É., 1998, «Villes et campagnes d'Égypte à l'aube du XX siècle : les temporalités divergentes des pays, des bourgs et des cités ». Urbanités arabes, Mélanges offerts à $B$. Lepetit, Coord. J. Dakhlia, Arles, Sindbad/Actes Sud.

Beinin J. \& Lockman Z., 1987, Workers on the Nile, Princeton University Press.

Delcroix M., 1922, « L'institution municipale en Égypte », L'Égypte contemporaine, nº 13.

Denis É., 2001, « L'après schéma directeur au Caire : Quand la ville déborde son enceinte », Villes en parallèle, $\mathrm{n}^{\circ}$ 30-31.

Denis É., 1995, « La mise en scène des ashwaiyyat. Premier acte Imbâba », Égypte Monde Arabe, nº 20.

Denis É. \& Bayoumi H., 1994, « Gamal Hamdan au Caire de l'Égypte », Lettre de l'Observatoire urbain du Caire contemporain, $\mathrm{n}^{\circ} 36$.

Doxiadis C., 1975, Ecumenopolis, the Inevitable City of the Future, Athènes, Athens Publishing Center.

El Kadi G., 1990, « Trente ans de planification urbaine au Caire », Revue Tiers Monde, $\mathrm{n}^{\circ} 121$.

El Kadi G., 1988, « Market Mechanisms and Spontaneous Urbanization in Egypt », International Journal of Urban and Regional Planning, vol. 12, $\mathrm{n}^{\circ} 1$.

Fanchette S., 1997, «Le Delta du Nil. Densités de population et urbanisation des campagnes », Fascicule de recherches, Urbama, Université de Tours, $\mathrm{n}^{\circ} 32$.

Hansen B., 1965, « Marginal Productivity Wage Theory and Subsistence Wage Theory in Egyptian Agriculture », Memo n547, Le Caire, Institute of National Planning.

Hamdân G., 1997, La personnalité de l'Égypte, 1967 pour la première édition (Dar al-Hilal, le Caire), 1980-1984 pour l'édition détaillée en 4 vol., 1997 pour la traduction française du volume de 1967 par le ministère égyptien de la Culture (série Prisme $n^{\circ} 1$ ).

Ireton F., 1998a, «Les trajectoires migratoires internes en Égypte d'après les recensements de 1976 et 1986 ", Fascicule de recherches, Tours, Urbama, CEDEJ-CERMOC, n 27.

Ireton F., 1998b, « L'immigration dans les cinq plus grandes villes d'Égypte 1907-1986 », ÉgypteMonde Arabe, $\mathrm{n}^{\circ} 32$.

Mabro R. \& Radwan siècle, 1976, The Industrialization of Egypt 1939-1973, Clarendon Press, Oxford, $276 \mathrm{p}$.

Mead D., 1967, Growth and Structural Change in the Egyptian Economy, R. D. Irwin Inc, Homewood, Illinois.

Owen R., 1981, The Middle East in the World Economy 1800-1914, Methuen \& Co, Londres.

Roussillon A., 1995, Entre réforme sociale et mouvement national, Le Caire, CEDEJ.

Sadowski Y., 1991, Political Vegetables. Businessman and Bureaucrat in the Development of Egyptian Agriculture, Brookings Institution, Washington.

Weber M., 1982, La ville, Paris, Aubier. 


\section{NOTES}

1.Pour Doxiadis, « l'écouménopolis » exprime le stade ultime de l'urbanisation de l'écoumène, c'est-à-dire le moment où la totalité de l'espace habité devient, par coalescence et inter connexion des unités de peuplement, une vaste ville.

2.Doxiadis a forgé le terme " d'épeiropolis » pour désigner ce stade intermédiaire sur le chemin de « l'écouménopolis ». L'image du rhizome est parfois utilisée pour caractériser ces étirements linéaires des systèmes de villes mais elle se réfère à une analyse différente qui postule le caractère aléatoire de leur évolution renvoyant à l'usage qu'en font Deleuze et Gattari pour rendre compte de l'expansion à distance, anarchique, imprévisible et non hiérarchisée (Mille plateaux, capitalisme et schizophrénie, éd. de Minuit, 1990). L'image de l'araignée renvoie évidemment à une organisation d'une tout autre nature, structurée et centralisée.

3.Le concept de « mégalopolis » établi par Jean Gottmann en 1957 désigne les vastes conurbations qui concentrent des fonctions de commandement majeures à l'échelle des plus grandes nations et du monde, et en particulier l'aire métropolitaine de la Côte est nord-américaine, de Boston à Washington en passant par New-York (« Megalopolis, or the Urbanization of the Northeastern Seabord », Economic Geography, 33, p. 189-200 ; 1962, Megalopolis, New York, Twenty Century Fund).

4. "L'homogénéité urbaine », chapitre 4, section 18, p. 212, 1981. Gamâl Hamdân, géographe mort en 1993 dans des conditions tragiques, s'est imposé comme le penseur de l'identité de l'Égypte, avec l'extension de son œuvre, d'abord publiée en un volume de 350 pages en 1967, après la défaite, puis en quatre volumes totalisants plus de 3200 pages édités entre 1980 et 1984. Il s'agit d'un modèle d'anti-géographie qui cherche à construire à la fois la centralité de l'Égypte à l'échelle planétaire et du monde arabe, comme de l'histoire de l'humanité, et une Égypte monolithique, gommant par tous les procédés possibles les nuances régionales pour construire une indéfectible homogénéité transhistorique garante de la puissance et justifiant la centralité immuable du pouvoir. Sont notamment utilisés une approche raciale faisant référence à des auteurs peu fréquentables et les despotismes oriental et hydraulique, compris comme une évidence. Il n'en reste pas moins que l'œuvre détaillée, très documentée, rend compte de la structuration du territoire égyptien. $C f$. la notice biographique rédigée par Mona Akouri (1993) ainsi que Denis et Bayoumi (1994).

5.La notion plutôt anglo-saxonne de urban bias a longtemps servi à stigmatiser le caractère négatif de l'urbanisation primatiale dans les pays dits du Sud, car captant la majorité des moyens pour une productivité faible et des effets déstructurants. Elle reste néanmoins utile pour désigner la pression sur les finances publiques et l'attractivité non moins cumulative sur les capacités d'investissement privées qu'a entrainées et entraîne toujours l'affirmation des capitales des États indépendants. À présent, les institutions internationales, telle la Banque mondiale, se sont davantage ralliées à l'idée libérale de la métropole comme moteur de la croissance ou motor engine, qui permet de justifier la polarisation de l'effort d'investissement et l'effacement des politiques de rééquilibrage régional dont les États n'ont de toute façon plus les moyens.

6.En 1939, l'Égypte est le dixième pays pour l'usage de fertilisants et le premier de ceux qui deviendront les pays du tiers-monde, au même niveau que l'Europe de l'Ouest, la Nouvelle-Zélande ou le Japon. Néanmoins, durant toute cette période, les rendements agricoles souffrent de problèmes de drainage consécutifs à l'extension du système d'irrigation que l'amendement chimique ne peut compenser. 
7.Pour une analyse de la « réforme sociale », $c f$. Roussillon en Égypte, voir l'ouvrage collectif édité par A. Roussillon, Entre réforme sociale et mouvement national, CEDEJ, $590 \mathrm{p}$. 8.Confirmant ce point, voici ce que relèvent les auteurs du schéma directeur de 1956 à propos de la situation socioprofessionnelle et l'apparent surpeuplement du Caire après la Seconde Guerre mondiale : " Nor should we omit the Second World War as one of the major factors for the acute situation. The allied armies stationed in Cairo provided additional opportunities for employment, new factories were opened, aerodromes were constructed and an intense commercial movement began. People from all trades and professions rushed to the city in search for better chances of labor, life, opulence or even pleasure. It was assumed that this concentration would be temporary, to be liquidated with the cessation of hostilities, however, it turned otherwise. Immigrants began to settle; they married, brought their dependents and induced others to follow suite, all to add to the acuteness of the situation. This caused terrific shortage of living accommodations, as during the war, all accommodations were provided by the Allied Armies Authorities or they were satisfied by living in single rooms, etc., but after they got married or brought their families along and the Allied Armies evacuated and deserted their camps, these laborers naturally needed accommodations to live in. » (Master Plan of Cairo, Ministry of Municipal \& Rural Affairs, Municipality of Cairo, Plan Commission, Presse de la Société orientale de publicité, 1957, p. 35-36).

9.P. O'Brien note qu'entre 1945 et 1957, si le produit industriel croît en moyenne de $6 \%$ par an et si la part de l'industrie passe de $7 \%$ à $10 \%$ du PNB, l'emploi industriel baisse de $9 \%$. La croissance de 2,2 \% indiquée pour la période intercensitaire 1947-1960 repose sur des données beaucoup plus agrégées qui incluent tout le secteur de l'auto-emploi manufacturier et de la micro-industrie des ateliers.

10.Jusqu'à présent, le destin de ces villes reste très incertain. Elles souffrent désormais des exigences de rapidité du commerce maritime entre les pôles de production asiatiques et l'Europe. Le Canal n'est plus qu'un tunnel où tout arrêt est une perte financière pour les compagnies maritimes. Dans ce contexte, le projet de port de redistribution de containers à Port-Sa îd à l'échelle de la Méditerranée orientale demeure incertain d'autant qu'il concurrence les ports de Beyrouth et de Dubaï. 11.Dans le budget de l'État 2000-2001, la région du Grand-Caire capte encore $31 \%$ de l'effort d'équipement public, auquel s'ajoute $51 \%$ du budget d'infrastructure pour les villes nouvelles. L'un des résultats de ces orientations est, qu'à présent la région métropolitaine dispose de $50 \%$ du réseau de collecteur d'égouts du pays. Depuis 20 ans, $78 \%$ des logements subventionnés construits dans les villes nouvelles l'ont été dans la périphérie du Grand-Caire (et, depuis 3 ans, $86 \%$ !).

12.Provoquant de graves crises de subsistance, les sécheresses sont, par exemple au Soudan mais aussi au Maroc, un facteur essentiel d'amplification et de non-tarissement de l'exode rural. Les études montrent clairement que ce qui s'envisageait dans un premier temps comme un déplacement saisonnier de survie vers la ville se transforme, la plupart du temps, en une migration définitive. 
INDEX

Mots-clés : géographie, Le Caire, géographie urbaine, métropole

\section{AUTEUR}

ERIC DENIS

Cedej 